\title{
Intrinsic long-range bond-order potential for carbon: Performance in Monte Carlo simulations of graphitization
}

\author{
J. H. Los and A. Fasolino \\ Theoretical Physics, NSRIM, University of Nijmegen, Toernooiveld, 6525 ED Nijmegen, The Netherlands
}

(Received 11 February 2003; published 31 July 2003)

\begin{abstract}
We propose a bond order potential for carbon with built-in long-range interactions. The potential is defined as the sum of an angular and coordination dependent short-range part accounting for the strong covalent interactions and a radial long-range part describing the weak interactions responsible, e.g., for the interplanar binding in graphite. The short-range part is a Brenner type of potential, with several modifications introduced to get an improved description of elastic properties and conjugation. Contrary to previous long-range extensions of existing bond order potentials, we prevent the loss of accuracy by compensating for the additional long-range interactions by an appropriate parametrization of the short-range part. We also provide a short-range bond order potential. In Monte Carlo simulations our potential gives a good description of the diamond to graphite transformation. For thin (111) slabs graphitization proceeds perpendicular to the surface as found in $a b$ initio simulations, whereas for thick layers we find that graphitization occurs layer by layer.
\end{abstract}

DOI: 10.1103/PhysRevB.68.024107

PACS number(s): 81.05.Uw

\section{INTRODUCTION}

In the past decade much progress has been made in the evolution of empirical potentials for covalent materials, in particular silicon and (hydro)carbon(s), for use in large scale simulations. Disregarding force field models, which are designed to give a good description close to equilibrium, in the literature we can distinguish various types of models: (i) Stillinger-Weber (SW) type potentials, ${ }^{1-4}$ (ii) Tersoff type bond order potentials (BOPs), ${ }^{5-8}$ (iii) embedded atom models (EAMs) ${ }^{9-11}$ and (iv) higher order bond order potentials derived from tight binding models (TBBOPs). ${ }^{12,13}$ Such models are meant to give a good description of the energy landscape for any possible realistic configuration characterized by the set of atomic positions $\left\{\mathbf{r}_{i}\right\}$.

Many empirical potentials have been designed and parametrized for silicon ${ }^{1,3,5,10,11}$ as a prototype of covalent materials. These models cannot straightforwardly be reparametrized for carbon. Since carbon is smaller than silicon, there is a stronger coupling between the free orbitals of undercoordinated neighboring atoms which results in a stronger $\pi$-bond formation and can lead to complicated hybridization situations. This requires an approach going beyond nearest neighbors, because the degree of conjugation of the bond between atom $i$ and $j$ crucially depends on the coordination of the neighbors of atoms $i$ and $j$. A reasonably good first order approximation of conjugation effects is included in the BOPs for hydrocarbons by Brenner and co-workers. ${ }^{7,8}$ For a more accurate description one should consider the hydrocarbon TBBOP of Pettifor and Oleinik. ${ }^{12,13}$ This model is more complex and computationally expensive, but it is still much more efficient than $a b$ initio calculations within the local density approximation (LDA) or tight-binding calculations. The TBBOP models also provided a more solid theoretical foundation for the EAM and the BOPs, ${ }^{12}$ which are essentially based on the second moment approximation of the total energy within a tight-binding formulation, and thus involve only nearest neighbors. Recently it was shown that such a close relationship also exists between the EAM model and the SW model. ${ }^{14}$

The starting point for our long-range $\mathrm{BOP}$ are the BOPs for carbon by Brenner. The most recent Brenner potential, the so-called reactive bond order (REBO) potential, ${ }^{8}$ which was not yet published when we started this work, combines the good properties of the two parametrizations of the earlier Brenner potentials I and II. ${ }^{7}$ Brenner I underestimates the isotropic elastic constants, whereas Brenner II gives too large interatomic distances. In addition, the REBO potential is supposed to give a better description of conjugation by an increased number of parameters, which are fitted to a larger data set. The Brenner potentials give a first order approximation to conjugation in an empirical way, including contributions beyond nearest neighbors. However, as all other models mentioned above, including the TBBOPs, with a typical cutoff radius between first and second nearest neighbors, the Brenner potentials describe only the strong covalent interactions and neglect long-range interactions. In particular, it does neither describe the relatively weak interplanar binding energy in graphite $\left(\sim 25 \mathrm{meV} / \mathrm{atom}^{15,16}\right.$ at an interplanar equilibrium distance of $\sim 3.35 \AA$ ), nor the much stronger interplanar interaction at shorter interplanar distances [see Fig. 2(b)] due to $\pi$-bond repulsion. For molecular dynamics (MD) or Monte Carlo (MC) simulations of diamond graphitization or of the formation of nested fullerenelike structures, long-range interactions play an important role and have to be taken into account. However, the problem is how to add these interactions to the Brenner potential without disturbing its nicely fitted properties, in particular the binding energy and lattice constants of the diamond and graphite structures, as well as the conjugation effects taken into account by Brenner's conjugation function $F^{c o n j}$. In the long-range extensions proposed in the literature so far, this problem has been addressed by switching off long-range interactions for "too close" atoms using a smooth cutoff. The switching functions 
are either based on distance, ${ }^{17,18}$ or else on neighbor connectivity. ${ }^{19}$ A combination of these two criteria has led to the so-called adaptive intermolecular REBO (AIREBO) potential. ${ }^{20}$ The philosophy of all these approaches is to add a long-range potential and use switching functions so that the short-range potential does not need to be reparametrized. However, this approach has its prices to be paid. In spite of the switching functions, the long range extension introduces a loss of accuracy. ${ }^{20}$ Moreover, the approaches based on the first criterion fail to describe the strong interplanar repulsion in graphite at short interplanar distances, i.e., distances of the order of the cutoff radius of the short-range part of the potential, where the long-range interactions are suppressed to avoid interference. The second, connectivity criterion, based on exclusion of long-range interactions between atoms which are connected as first, second or third neighbors, has no good physical justification. It gives rise to peculiar indirect interactions between particles. In fact, in Monte Carlo simulations of graphitization based on a long-range extended potential based on this criterion, we find unrealistic structural defects, as we will show in Sec. IV.

The long-range carbon bond order potential (LCBOP) we propose here is based on an alternative approach: we exclude long-range interactions only for nearest neighbors and parametrize the short-range part of the potential in such a way that the combined potential yields the correct properties, i.e., lattice constants, binding energies, elastic properties, and conjugation effects. Instead of the Lennard-Jones potential used in Refs. 19 and 20, our long-range potential is a Morselike potential which is based on a best fit of the interlayer interaction energy in graphite, calculated by the LDA, ${ }^{21}$ for a range of interplanar distances. The functional form of the short-range part of our potential contains a number of modifications as compared to the Brenner potential, enabling, among other things, a better fit of the shear elastic constant for diamond. We also propose a different interpolation scheme for the conjugation correction, relevant for mixed coordinated environments. This interpolation scheme is justified in terms of a simple model. Together with that of the LCBOP we also give a parametrization of our short-range potential without long-range interactions, which we denote as CBOP. This short-range version, which is computationally more efficient than the LCBOP, can be used in situations where long-range interactions are not so relevant.

As a test, we have used the LCBOP in Monte Carlo simulations of diamond graphitization, i.e., the transformation of diamond to graphite, observed experimentally at temperatures varying between $1300 \mathrm{~K}$ (Ref. 22) and $1800 \mathrm{~K}^{23}$ Usually graphitization starts at the (111) surface, the dominant face in the morphology of diamond. Graphitization has also been studied theoretically by ab initio molecular dynamics (AIMD) simulations ${ }^{24-26}$ for relatively small samples, typically with not more than 200 atoms. The AIMD simulations show the formation of perfect graphitic planes from a (2 $\times 1)$ Pandey reconstructed (111) slab containing six bilayers at temperatures varying between $2500 \mathrm{~K}$ (Ref. 24) and 3500 $\mathrm{K},{ }^{26}$ depending on the details of the thermostats used and the run settings. For a nonreconstructed slab of the same size the transition temperature was found to be much lower. ${ }^{26}$ In all the AIMD simulations for thin unsaturated slabs, after the nucleation of graphitic islands at the surface, the graphitization first proceeds into the slab, perpendicular to the surface plane, before any full graphitic plane is formed. In our simulations, we find the same behavior for thin slabs. However, for thicker slabs we find a layer by layer mechanism.

The details of our long-range bond order potential for carbon are described in Sec. II. In Sec. III the parameters and various performance data for the LCBOP and CBOP are given. Apart from binding energies and interatomic distances, the performance data also include elastic properties, surface reconstructions and the energy barrier for the transformation from bulk diamond to bulk rhombohedral graphite. In Sec. IV we illustrate the performance of the LCBOP in Monte Carlo simulations of graphitization for various geometries. Conclusions and perspectives are given in Sec. V.

\section{LONG RANGE BOND ORDER POTENTIAL FOR CARBON, LCBOP}

The total binding energy $E_{b}$, according to the LCBOP, is written as a sum of pair terms:

$$
E_{b}=\frac{1}{2} \sum_{i, j}^{N} V_{i j}^{t o t}=\frac{1}{2} \sum_{i, j}^{N}\left(f_{c, i j} V_{i j}^{S R}+S_{i j} V_{i j}^{L R}\right),
$$

where the total pair interaction $V_{i j}^{\text {tot }}$ is the sum of a shortrange part, $f_{c, i j} V_{i j}^{S R}$, describing the covalent interaction, and a long-range part $S_{i j} V_{i j}^{L R}$. The function $f_{c, i j} \equiv f_{c}\left(r_{i j}\right)$ is a smooth cutoff function, specified below, and $S_{i j}$ is a switching function, to exclude first neighbors, given by

$$
S_{i j}=1-f_{c, i j}
$$

The short-range part is written as

$$
V_{i j}^{S R}=V_{R}\left(r_{i j}\right)-B_{i j} V_{A}\left(r_{i j}\right),
$$

where $V_{R}$ and $V_{A}$ are repulsive and attractive radial pair potentials and $B_{i j}$ is the bond order, containing many body effects. We found that a good simultaneous fit of binding energies, lattice constants, and isotropic elastic properties for the various polytypes of carbon could be obtained with the following forms for $V_{R}$ and $V_{A}$ :

$$
V_{R}(r)=A \exp (-\alpha r)
$$

and

$$
V_{A}(r)=B_{1} \exp \left(-\beta_{1} r\right)+B_{2} \exp \left(-\beta_{2} r\right) .
$$

As cutoff function we have adopted the one used by Justo for $\mathrm{EDIP}^{3}$ a SW type of potential for silicon:

$$
f_{c}(x)=\Theta(-x)+\Theta(x) \Theta(1-x) \exp \left(\frac{\gamma x^{3}}{x^{3}-1}\right),
$$


with $x=\left(r-r_{1}\right) /\left(r_{2}-r_{1}\right)$, and where $\Theta(x)$ is the Heavyside step function. The function $f_{c}$ is continuous up to the second derivative at $r=r_{1}$ and in all its derivatives at the cut-off radius $r_{2}$. It yields a smoother cut-off than the cosine function used by Brenner and Tersoff, which has nonzero second derivatives at $r_{1}$ and $r_{2}$. The extra parameter $\gamma$ has been used to optimize the shape of the energy barrier for the diamond to graphite transformation, as described in Sec. III. Following Brenner, the bond order $B_{i j}$ is taken as

$$
B_{i j}=\frac{1}{2}\left[b_{i j}+b_{j i}+F^{c o n j}\left(N_{i j}, N_{j i}, N_{i j}^{c o n j}\right)\right],
$$

where $b_{i j}$ is the angular dependent part of the bond order and $F^{c o n j}$ takes conjugation effects into account. The angular dependent part is written as

$$
b_{i j}=\left(1+\sum_{k \neq i, j} f_{c}\left(r_{i k}\right) G\left(\cos \theta_{i j k}\right) H\left(\delta r_{i j k}\right)\right)^{-\delta},
$$

where the summation runs over all neighbors $k(\neq j)$ of $i, \theta_{i j k}$ is the bond angle between the bonds $i j$ and $i k$, and $\delta r_{i j k}$ is the difference in bond distance between these two bonds, namely, $\delta r_{i j k}=r_{i j}-r_{i k}$. Following the result of Abell, ${ }^{27}$ we take $\delta=1 / 2$. For the angular function $G\left(\cos \theta_{i j k}\right)$, shown in Fig. 1(a), a spline is used, as for the REBO potential, based on its values at the discrete points corresponding to regular lattices. More details are given in Sec. III. The function
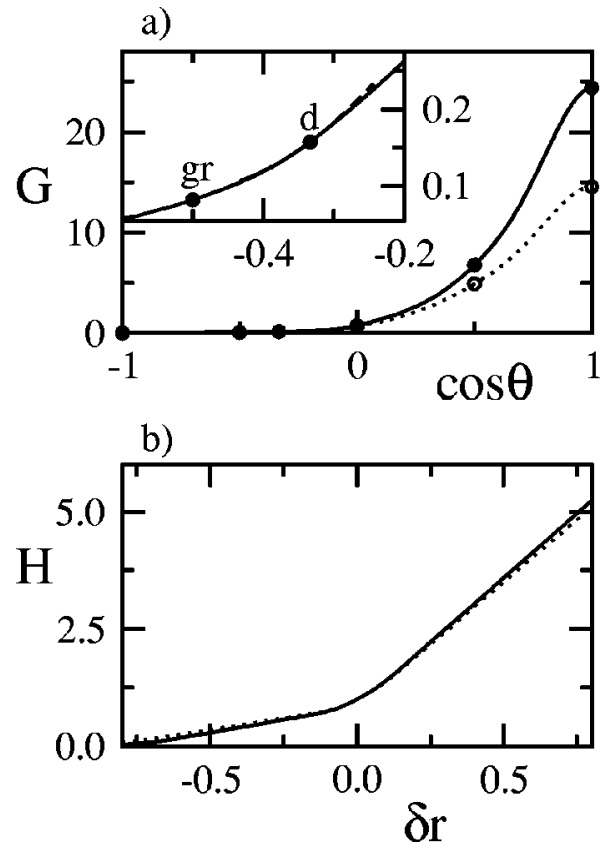

FIG. 1. The bond order functions $G(\cos \theta)(1 \mathrm{a})$ and $H(\delta r)(1 \mathrm{~b})$ for the LCBOP (solid line) and the CBOP (dotted line). The dashed lines in the inset of the upper graph, which contains a zoom of $G$ around the bond angles for graphite (gr) and diamond (d), represent the quadratic curvatures required to fit the elastic constants given in Table II. The vertical axis of the inset is labeled on the right-hand side.

$H\left(\delta r_{i j k}\right)$, not present in Brenner's potentials for carbon, is introduced to optimize elastic properties, surface properties and the energy barrier for the diamond to graphite transformation. We propose the following form:

$$
H(x)=\left\{\begin{array}{l}
H_{1}(x)=L\left(1+\kappa(x+d)\left(\frac{1}{1+[\kappa(x+d)]^{10}}\right)^{1 / 10}\right), \quad x<-d \\
H_{2}(x)=1+C_{1} x+\frac{1}{2} C_{1}^{2} x^{2}+C_{4} x^{4}+C_{6} x^{6}, \quad-d \leqslant x \leqslant d \\
H_{3}(x)=R_{0}+R_{1}(x-d), \quad x>d,
\end{array}\right.
$$

where $d$ is a fit parameter. This forms was established as follows. Good elastic properties could be obtained assuming a simple exponential $H(x)=\exp \left(C_{1} x\right)$ for small $x$. This implies first and second derivatives equal to $C_{1}$ and $C_{1}^{2}$ at $x$ $=0$. An improved description of Pandey's $(2 \times 1)$-reconstructed (111) surface for diamond required a certain amount of non-parabolicity within the interval $[-d, d]$, which is introduced by a fourth order term with a coefficient $C_{4}$. Outside this interval a basically linear behavior was assumed, with a smooth tail tending to zero from above for negative $x$. This behavior for $|x|>d$ allows a reasonable description of the reaction path of the diamond to graphite transformation (see Sec. III). The function $H$ is completely fixed by only three parameters, namely $d, C_{1}$ and $C_{4}$. The remaining parameters, i.e. $L, \kappa, C_{6}, R_{0}$, and $R_{1}$, follow from the continuity of $H$ up to its second derivative at $x= \pm d$. In particular, $C_{6}$ follows directly from $d^{2} H_{2} /\left.d x^{2}\right|_{x=d}=d^{2} H_{2} /\left.d x^{2}\right|_{x=-d}=0$. Note that by construction $d^{2} H_{1} /\left.d x^{2}\right|_{x=-d}=d^{2} H_{3} /\left.d x^{2}\right|_{x=d}=0$. The function $H(x)$ is shown in Fig. 1(b) for the LCBOP and CBOP.

The coordination of atom $i, N_{i}$, is defined as

$$
N_{i}=\sum_{k} f_{c, i k} .
$$

The argument $N_{i j}$ of the conjugation function $F^{c o n j}$ is defined as 


$$
N_{i j}=\min \left(3, \sum_{k \neq j} f_{c, i k}\right)=\min \left(3, N_{i}-f_{c, i j}\right),
$$

where $\min (x, y)$ stands for the minimum of $x$ and $y$. As will be clarified below, we define the third argument of $F^{c o n j}$, $N_{i j}^{c o n j}$, as a number within the interval $[0,1]$ by

$$
N_{i j}^{c o n j}=\frac{\left(N_{i j}+1\right)\left(N_{j i}+1\right)\left(N_{i j}^{e l}+N_{i j}^{e l}\right)-4\left(N_{i j}+N_{j i}+2\right)}{N_{i j}\left(3-N_{i j}\right)\left(N_{j i}+1\right)+N_{j i}\left(3-N_{j i}\right)\left(N_{i j}+1\right)+\epsilon},
$$

where $\epsilon$ is a very small, positive number, added to prevent the singularities occurring for $N_{i j}=N_{j i}=0$ or $N_{i j}=N_{j i}=3$, where the numerator in Eq. (12) also vanishes, as can be verified using the definitions below. $N_{i j}^{e l}$ gives the contribution of electrons from atom $i$ to the bond $i j$. We define it as

$$
N_{i j}^{e l}=\frac{4-M_{i j}}{N_{i j}+1-M_{i j}},
$$

where $M_{i j}$ is given by

$$
M_{i j}=\min \left(3, \tilde{M}_{i j}\right),
$$

with $\widetilde{M}_{i j}$ the number of neighbors $k \neq j$ of atom $i$ which have a coordination $\geqslant 4$. $\tilde{M}_{i j}$ is defined as

$$
\tilde{M}_{i j}=\sum_{k \neq i, j} f_{c, i k} F\left(x_{i k}\right),
$$

where $x_{i k}=N_{k}-f_{c, i k}$ and $F\left(x_{i k}\right)$ is given by

$$
\begin{aligned}
F\left(x_{i k}\right)= & \Theta\left(x_{i k}-3\right)+\frac{1}{2} \Theta\left(x_{i k}-2\right) \Theta\left(3-x_{i k}\right) \\
& \times\left(1-\cos \left[\pi\left(x_{i k}-2\right)\right]\right) .
\end{aligned}
$$

We note that our $F\left(x_{i k}\right)$ is one minus Brenner's $F\left(x_{i k}\right)$.

Our definition of $N_{i j}^{c o n j}$ by Eq. (12) is completely different from that of Brenner and requires some explanation, which we give in terms of a simple model. For convenience we will restrict ourselves to situations with integer coordinations.

Consider the case $N_{i} \leqslant 4 \forall i$. If a neighbor $k \neq j$ of $i$ has a coordination 4 , bond $i k$ is single since all four $s, p$ orbitals of atom $k$ are involved in $\sigma$ bonds, so that there are no free orbitals available to form $\pi$ bonds with atom $i$. So $M_{i j}$ represents the number of electrons of atom $i$ involved in single $i k(\neq i j)$ bonds, containing one electron from atom $i$ and one from atom $k$, and $N_{i j}^{e l}$ as defined by Eq. (13) gives the (fractional) number of electrons that are left for the remaining bonds of atom $i$. Consider the situation of Fig. 2, where atoms $i$ and $j$ have both coordination 3. According to Eq. (13), a coordination situation $\left(N_{k_{1}}, N_{k_{2}}, N_{l_{1}}, N_{l_{1}}\right)=(4,3,3,3)$ yields $N_{i j}^{e l}=3 / 2$ and $N_{j i}^{e l}=4 / 3$. The average electronic contribution to bond $i j$ in this case is thus $\bar{N}_{i j}^{e l}=\left(N_{i j}^{e l}+N_{j i}^{e l}\right) / 2$ $=4 / 3+1 / 12$, i.e., still rather close to the "graphitic value" $4 / 3$. In contrast, for coordinations $\left(N_{k_{1}}, N_{k_{2}}, N_{l_{1}}, N_{l_{1}}\right)$ $=(4,4,4,4)$, bond $i j$ is a double bond and, indeed, we find $\bar{N}_{i j}^{e l}=2$. For general coordination numbers $\left(N_{i j}, N_{j i}\right), \bar{N}_{i j}^{e l}$ is

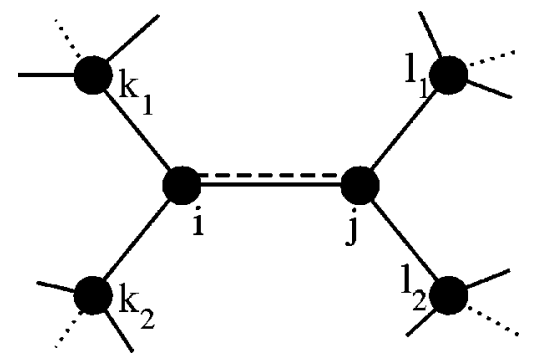

FIG. 2. Sketch of three fold coordinated neighbors $i$ and $j$.

bounded by a minimum and a maximum value, $\min \left(\bar{N}_{i j}^{e l}\right)$ and $\max \left(\bar{N}_{j i}^{e l}\right)$ respectively. For instance, for $\left(N_{i j}, N_{j i}\right)=(3,3)$, $\min \left(\bar{N}_{i j}^{e l}\right)=4 / 3$ and $\max \left(\bar{N}_{j i}^{e l}\right)=2$. It is reasonable to assume a linear dependence of $F^{c o n j}$ on $\bar{N}_{i j}^{e l}$, which can be accomplished by defining $N_{i j}^{c o n j}$ as a number within the interval $[0,1] \quad$ by $\quad N_{i j}^{c o n j}=\left[\bar{N}_{i j}^{e l}-\min \left(\bar{N}_{i j}^{e l}\right)\right] /\left[\max \left(\bar{N}_{j i}^{e l}\right)-\min \left(\bar{N}_{i j}^{e l}\right)\right]$, which, after some algebra, leads to Eq. (12).

For $N_{i} \geqslant 4$, implying $N_{i j}=3$, the bond $i j$ is a single bond, independent of the coordinations of the neighbors $k(\neq j)$. Therefore we assume that $F^{c o n j}\left(N_{j i} \geqslant 3, N_{j i}, 0\right)$ $=F^{c o n j}\left(3, N_{j i}, 0\right)=F^{c o n j}\left(3, N_{j i}, 1\right)$, as for the Brenner potentials. Furthermore, due to symmetry, $F^{c o n j}\left(n, m, N_{i j}^{c o n j}\right)$ $=F^{c o n j}\left(m, n, N_{i j}^{c o n j}\right)$.

As already mentioned we assume that $F^{c o n j}$ depends linearly on $N_{i j}^{c o n j}$, implying

$$
\begin{aligned}
F^{c o n j}\left(N_{i j}, N_{j i}, N_{i j}^{c o n j}\right)= & \left(1-N_{i j}^{c o n j}\right) F_{0}^{c o n j}\left(N_{i j}, N_{j i}\right) \\
& +N_{i j}^{c o n j} F_{1}^{c o n j}\left(N_{i j}, N_{j i}\right),
\end{aligned}
$$

where $F_{N_{i j}^{c o n j}}^{c o n j}\left(N_{i j}, N_{j i}\right) \equiv F^{c o n j}\left(N_{i j}, N_{j i}, N_{i j}^{c o n j}\right)$. To deal with noninteger coordinations, we propose a computationally efficient interpolation for the bivariable functions $F_{0}^{c o n j}$ and $F_{1}^{c o n j}$, yielding continuity up to the first derivatives with respect to the arguments $N_{i j}$ and $N_{j i}$. This interpolation, described in detail in the Appendix, makes the computation of $F^{c o n j}$ relatively easy and considerably more efficient as compared to the tricubic spline proposed by Brenner, without losing quality.

The long-range pair potential $V_{i j}^{L R}$ is constructed by making a best fit of an $a b$ initio LDA calculation of the interplanar interaction energy in hexagonal graphite, $E_{l}\left(d_{l}\right)$, as a function of the interplanar distances $d_{l}$ beyond $2 \AA^{21}$ The $a b$ initio result is represented by the crosses in Fig. 3(b). In our description, the interplanar interaction energy is supposed to be equal to the sum over all pair interactions between particles in different layers:

$$
E_{l}\left(d_{l}\right)=\frac{1}{2} \sum_{i}^{\prime} \sum_{j}^{\prime} f_{c, i j}^{L R} V_{i j}^{L R}\left(r_{i j}\right)
$$

where the sum over $i$ runs over the atoms within one unit cell and the sum over $j$ runs over all atoms belonging to graphitic planes different from that to which atom $i$ belongs. The cutoff function $f_{c, i j}^{L R}$, added to switch off the tail of the longrange interactions in a range where these interactions are 

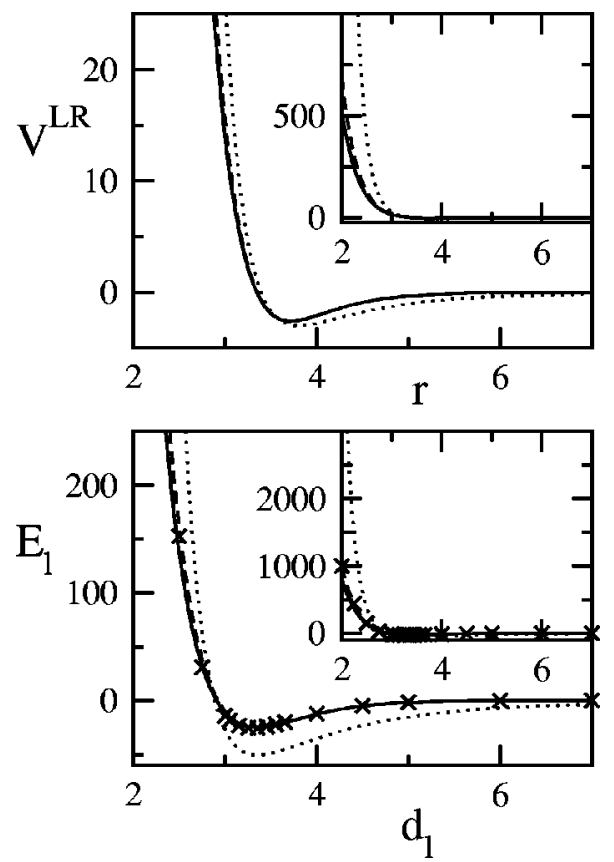

FIG. 3. (a) Various long-range pair potentials $V^{L R}$ (in $\mathrm{meV}$ ) as a function of the interatomic distance $r$ (in $\AA$ ) and (b) the corresponding interlayer interaction energies $E_{l}$ (in $\mathrm{meV}$ ) in graphite as a function of the interlayer distance $d_{l}$ (in $\AA$ ). The crosses in the bottom graph represent the results from an $a b$ initio LDA calculation. The solid and dashed lines represent the data assuming the double Morse potential [Eq. (19)] resulting from a best fit with and without counting the short-range contribution beyond $2 \AA$ respectively. For comparison, the dotted lines are the data for the Lennard Jones potential used in Ref. 19. The insets represent the same data on a larger energy scale.

already very weak, falls off from 1 to 0 between $r_{1}^{L R}$ $=5.5 \AA$ and $r_{2}^{L R}=6 \AA$ by $f_{c}^{L R}(r)=(1+\cos [2 \pi(r-5.5)]) / 2$. A good fit of the LDA data could be obtained by assuming the following double Morse potential for $V^{L R}(r)$ :

$$
V^{L R}(r)=\theta\left(r_{0}-r\right) V_{1}^{M}(r)+\theta\left(r-r_{0}\right) V_{2}^{M}(r),
$$

where $V_{i}^{M}(i=1,2)$ are ordinary Morse functions plus a shift

$$
V_{i}^{M}(r)=\epsilon_{i}\left(e^{-2 \lambda_{i}\left(r-r_{0}\right)}-2 e^{-\lambda_{i}\left(r-r_{0}\right)}\right)+v_{i} .
$$

This form allows the steepness of the potential on both sides of the minimum at $r=r_{0}$ to be adjusted independently. The two Morse functions are connected continuously up to the second derivative in $r=r_{0}$, implying $\epsilon_{1}=\epsilon_{2} \lambda_{2}^{2} / \lambda_{1}^{2}$ and $v_{1}$ $=\epsilon_{1}-\epsilon_{2}$ with $v_{2}=0$. In Fig. 3(a), $V^{L R}(r)$ is shown on two different energy scales and compared to the Lennard-Jones potential used in Ref. 19. Since the cutoff for the short-range part of the LCBOP is equal to $2.2 \AA$, there is a short-range contribution to the interlayer energy for $d_{l}<2.2 \AA$. The full and dashed lines in Fig. 3, which are very close, result from a best fit of the LDA interlayer energy with and without the short-range contribution, respectively. The latter pair potential is used in our simulations with an extended REBO potential, which has a short-range cut-off radius of $2 \AA$. We will come back to this in Sec. IV.
The resulting interplanar interaction energies for each of these potentials are shown in Fig. 3(b). Our long-range potential yields a very good fit of the LDA data. The resulting compressibility in the direction perpendicular to the layers is $5.48 \times 10^{-3} \AA^{3} / \mathrm{meV}$ which compares reasonably well with the experimental value $4.33 \times 10^{-3} \AA^{3} / \mathrm{meV}^{28,29}$ Figure $3(\mathrm{~b})$ clearly demonstrates that the Lennard-Jones potential yields a much too strong interplanar repulsion, with $E_{l} \simeq 5.4 \mathrm{eV}$ at $d_{l}=2 \AA$, as a direct consequence of the strong pair repulsion at short distances. This means that the Lennard-Jones potential would imply too high barriers for bond breaking and formation, when used as extensions of the short-range potential. Although our long-range potential yields a much weaker core repulsion, the repulsive interactions between second and third neighbors at distances $\sim 2.52$ and $\sim 2.96 \AA$ in diamond are still $\sim 100$ and $\sim 17 \mathrm{meV}$, respectively. Note that, for the contribution to the total binding energy, these numbers have to be multiplied by the number of second and third neighbors respectively. The philosophy of the LCBOP is to compensate for this additional repulsion between second and third neighbors by a stronger attraction between first neighbors, achieved by an appropriate parametrization of the shortrange part of the potential.

\section{FITTING PROCEDURE, PARAMETERS AND PROPERTIES}

The fitting procedure is performed in steps and iteratively. We assume an initial constant function $H=1$, and fit the radial part and the angular function $G$. Next, the parameters of the function $\mathrm{H}$ are determined by optimizing the elastic constants for uniaxial compression and shear for diamond ${ }^{30}$ and graphite, ${ }^{31}$ the distance between first and second bi-layer in the $(2 \times 1)$-Pandey-reconstructed (111)-surface of diamond $^{32}$ and the energy barrier for the graphite to diamond transformation. ${ }^{33}$ These steps are repeated until convergence is achieved. Finally, the conjugation correction matrices ( $F^{c o n j}$ at integer values of the arguments) are fitted.

First of all, we have to give a definition of bond energies within our potential form, which is applicable both to regular lattice bonding and to nonlattice bonding, and suitable for being fitted to the reference values, i.e., bond energies derived either from experimental data or from ab initio calculations. In presence of a long-range potential this requires some care. For a regular lattice with integer coordination $Z$ the approach is straightforward. Assuming $f_{c, i j}=1$ for near-

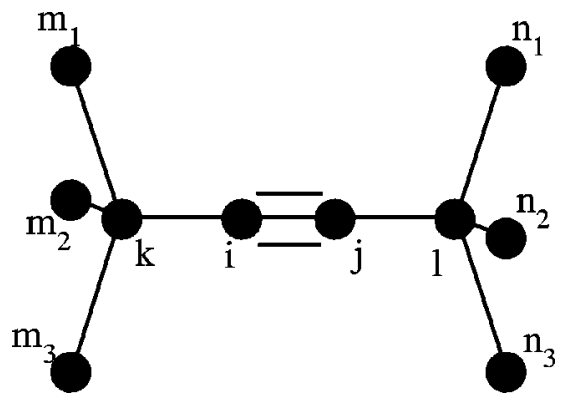

FIG. 4. Sketch of the configuration yielding a triple bond between atoms $i$ and $j$. 
TABLE I. Bond distances in $\AA$ (Ia), bond energies in eV (Ib) and stretching force constants in $\mathrm{eV} / \AA^{2}$ (Ic) for regular lattices, the dimer bond (di) and the triple bond (tb), yielding coordination $Z$, according to the LCBOP and CBOP, compared with the reference values, used as fitting data, and the values according to the Brenner I and the REBO potential. The reference values are taken from Refs. 7,8 and 42. The binding energy for graphite according to the LCBOP is for a sheet and does not include interlayer bonding energy. Adding the interlayer energy $(\sim 0.025 \mathrm{eV})$ leads to approximately equal total binding energies of $7.374 \mathrm{eV}$ for graphite according to the LCBOP and CBOP.

\begin{tabular}{|c|c|c|c|c|c|}
\hline $\mathrm{Z}$ & Ref. & LCBOP & CBOP & Brenner I & REBO \\
\hline \multicolumn{6}{|c|}{ (a) } \\
\hline 1 (di) & 1.315 & 1.315 & 1.315 & 1.315 & 1.326 \\
\hline $2(\mathrm{ch})$ & 1.330 & 1.325 & 1.325 & 1.325 & 1.332 \\
\hline $2(\mathrm{tb})$ & 1.200 & 1.200 & 1.200 & 1.196 & 1.206 \\
\hline 3 (gr) & 1.420 & 1.420 & 1.420 & 1.419 & 1.420 \\
\hline $4(d)$ & 1.544 & 1.544 & 1.544 & 1.541 & 1.544 \\
\hline $6(\mathrm{sc})$ & 1.765 & 1.803 & 1.843 & 1.833 & 1.875 \\
\hline $12(\mathrm{fcc})$ & 2.170 & 2.021 & 2.139 & 2.415 & 2.253 \\
\hline \multicolumn{6}{|c|}{ (b) } \\
\hline 1 (di) & 3.163 & 3.105 & 3.135 & 3.163 & 3.105 \\
\hline $2(\mathrm{ch})$ & 6.175 & 6.106 & 6.124 & 6.177 & 6.117 \\
\hline $2(\mathrm{tb})$ & 8.424 & 8.524 & 8.324 & 8.424 & 8.514 \\
\hline 3 (gr) & 7.374 & 7.349 & 7.374 & 7.377 & 7.395 \\
\hline $4(d)$ & 7.349 & 7.349 & 7.349 & 7.346 & 7.370 \\
\hline $6(\mathrm{sc})$ & 4.689 & 4.687 & 4.686 & 5.453 & 4.781 \\
\hline $12(\mathrm{fcc})$ & 2.759 & 2.757 & 2.758 & 2.683 & 2.782 \\
\hline \multicolumn{6}{|c|}{ (c) } \\
\hline $2(\mathrm{ch})$ & 59.67 & 61.33 & 61.83 & 27.80 & 59.54 \\
\hline $2(\mathrm{tb})$ & 99.86 & 99.04 & 98.69 & 37.91 & 98.99 \\
\hline $3(\mathrm{gr})$ & 43.57 & 43.71 & 44.07 & 22.13 & 43.56 \\
\hline $4(d)$ & 29.52 & 29.27 & 29.27 & 16.53 & 29.52 \\
\hline
\end{tabular}

est neighbors, the total bond energy $V_{i j}^{\text {tot }}[\mathrm{Eq} .(1)]$, can be defined as

$$
V_{i j}^{t o t}=V_{i j}^{S R}+2 \hat{E}_{i}^{L R}
$$

where $\hat{E}_{i}^{L R}$ is the long-range energy per atom "per shortrange bond" defined as

$$
\hat{E}_{i}^{L R}=\frac{E_{i}^{L R}}{Z}=\frac{1}{2 Z} \sum_{j} S_{i j} V_{i j}^{L R},
$$

where the sum runs over all atoms. The term $2 \hat{E}_{i}^{L R}$ in Eq. (21) can be written as the sum of two terms, namely, $2 \hat{E}_{i}^{L R}$ $=\hat{E}_{i j}^{L R}+\hat{E}_{j i}^{L R}$ where $\hat{E}_{i j}^{L R}$ contains all the long-range interactions of atom $i$ with the "branch" of atom $j$, i.e. the neighbors $l(\neq i)$ of atom $j$ and the subsequent neighbors of atom $l(\neq j)$ and so on, and $\hat{E}_{j i}^{L R}$ contains all the interactions of $j$ with the "branch" of atom $i$. This concept is transferable to nonlattice bonding types where an $i j$ bond forms the only connection between two separate branches, such as the triple
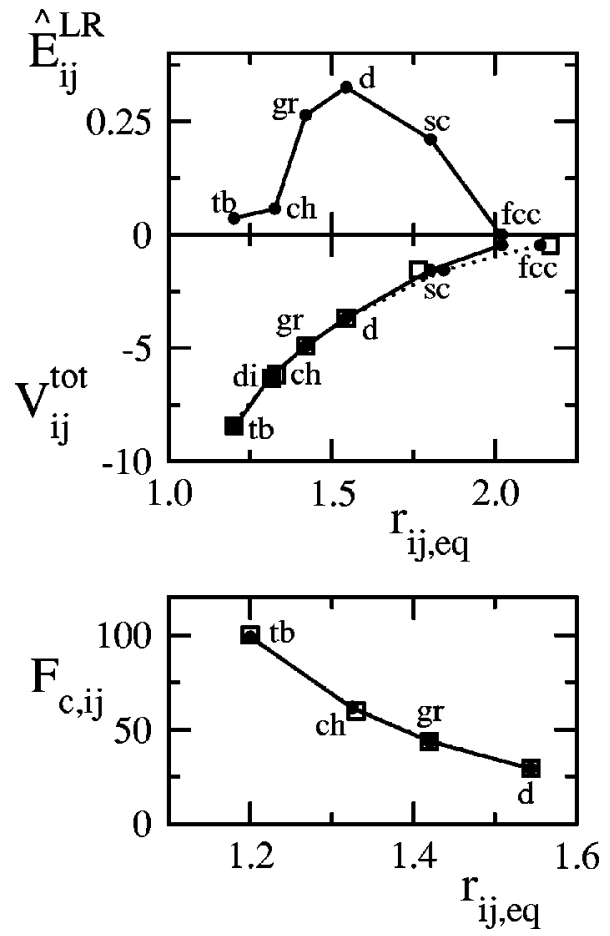

FIG. 5. The total bond energy $V_{i j}^{\text {tot }}$ (in eV) as defined by Eq. (21), the long-range contribution $\hat{E}_{i j}^{L R}$ (in eV) to $V_{i j}^{\text {tot }}$ as defined by Eq. (22) and the stretching force constants $F_{c, i j}$ (in $\mathrm{eV} / \AA^{2}$ ) for various regular lattices, the dimer (di) and the triple bond (tb) for the LCBOP and the CBOP, both represented by solid dots, as a function of the corresponding equilibrium nearest neighbor distance $r_{i j, e q}$ (in $\AA$ ). The open squares represent the reference values in Table I. The lines are guides to the eye. The solid and dashed lines, mostly very close, are for the LCBOP and CBOP respectively. Note the scale difference between the positive and negative energy axis in the upper graph.

bond situation sketched in Fig. 4. It is easy to verify for the cluster in Fig. 4 that with this 'branch' subdivision of the long-range contribution, the sum over all $V_{i j}^{t o t}$ for each nearest neighbor pair $i j$, contains precisely all the interactions within the cluster, i.e., it is equal to the total binding energy of the cluster, as it should be. The cluster of Fig. 4 was used to fit the triple bond properties. For this purpose a rigid and ideal tetrahedral nearest neighbor surrounding for the atoms $k$ and $l$ was assumed with all neighbor distances equal to the single bond distance in diamond, $1.544 \AA$. We checked that the effect of relaxations within the branches is small enough to be neglected.

TABLE II. Elastic constants (in $\mathrm{eV} / \AA^{3}$ ) for graphite (gr) and diamond (d) according to various potentials, compared with the reference data used in the fitting procedure. The reference values labeled by $*$ and $* *$ are taken from Refs. 30 and 31 , respectively.

\begin{tabular}{llcccc}
\hline \hline & Ref. & LCBOP & CBOP & Brenner I & REBO \\
\hline$c_{11}(\mathrm{gr})$ & $6.616^{*}$ & 6.547 & 6.570 & 3.466 & 6.600 \\
$c_{66}(\mathrm{gr})$ & $2.746^{*}$ & 2.780 & 2.773 & 1.567 & 2.835 \\
$c_{11}(\mathrm{~d})$ & $6.718^{* *}$ & 6.718 & 6.718 & 2.157 & 6.714 \\
$c_{44}(\mathrm{~d})$ & $3.604 * *$ & 3.604 & 3.604 & 1.719 & 4.499 \\
\hline \hline
\end{tabular}


TABLE III. The parameters of the LCBOP and CBOP. A, $B_{1}$ and $B_{2}$ are in $\mathrm{eV} ; v_{1}, \epsilon_{1}$ and $\epsilon_{2}$ are in meV; $r_{1}, r_{2}, d, r_{0}, r_{1}^{L R}$ and $r_{2}^{L R}$ are in $\AA ; \alpha, \beta_{1}, \beta_{2}, C_{1}, \kappa, R_{1}, \lambda_{1}$, and $\lambda_{2}$ are in $\AA^{-1} ; C_{4}$ is in $\AA^{-4} ; C_{6}$ is $\AA^{-6} ; \gamma, L$, and $R_{0}$ are dimensionless. The spline of the angular function $G$ is based on the data in Table II(b). (c) contains the $(4 \times 4)-F_{N_{i j}^{c o n j}}^{c o n j}$ matrices for $N_{i j}^{c o n j}=0,1$. The $(0,0)$ elements are given in left upper corners.

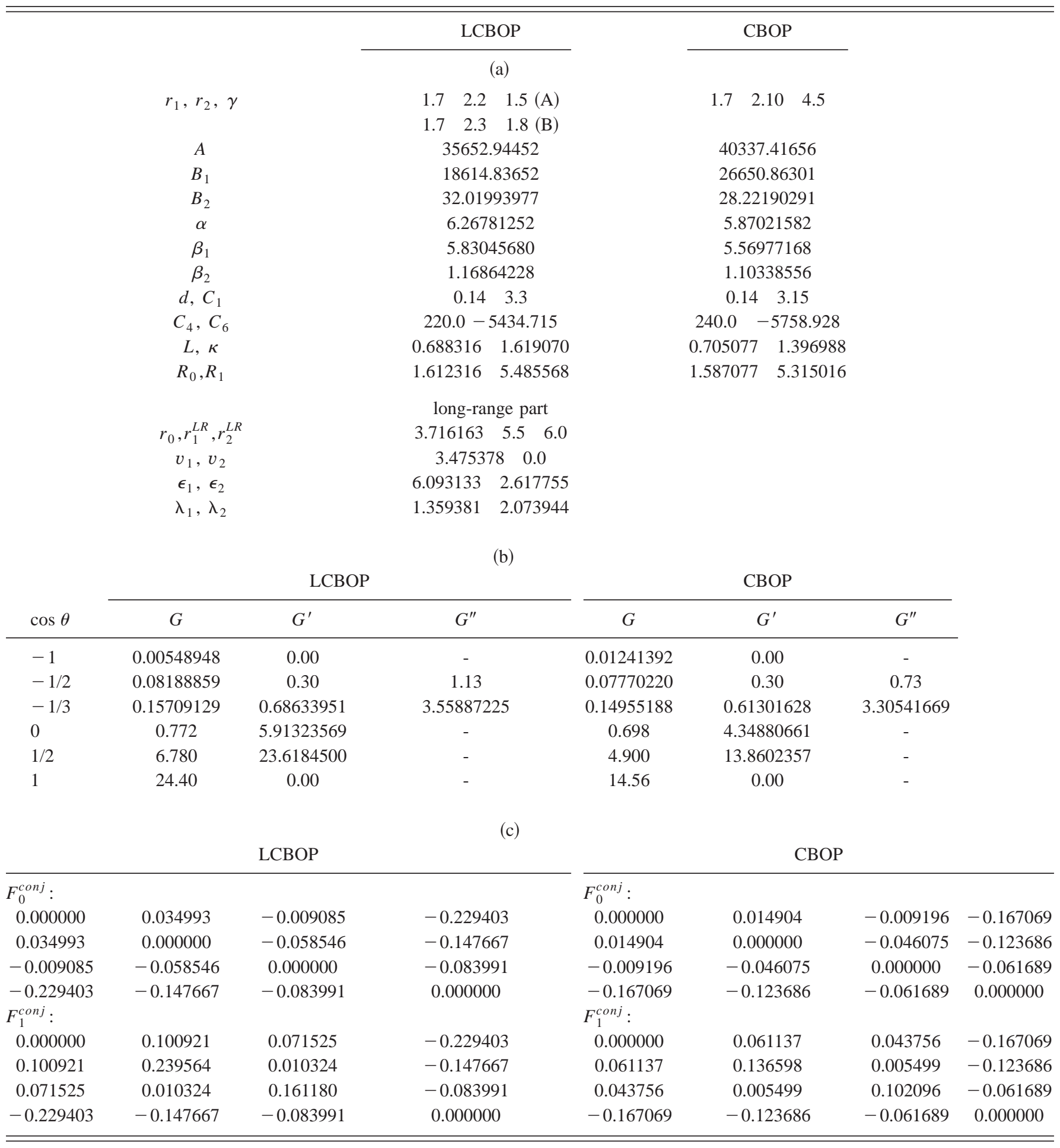

For regular lattices, the stretching force constants $F_{c, i j}$ are calculated by assuming an isotropic deformation. For the triple bond, the stretching force constant is calculated by an infinitesimal variation of only the triple bond distance, i.e., assuming rigid $i$ and $j$ "branches."
The bond distances, binding energies and stretching force constants, resulting from the radial part of the fitting procedure, are listed in Tables I(a), I(b), and I(c) respectively, together with those according to the Brenner I and the REBO potential. Globally the performances of the LCBOP and 


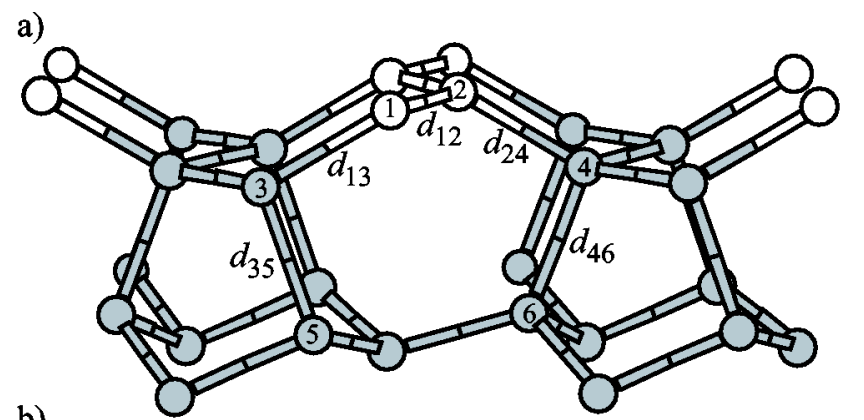

b)

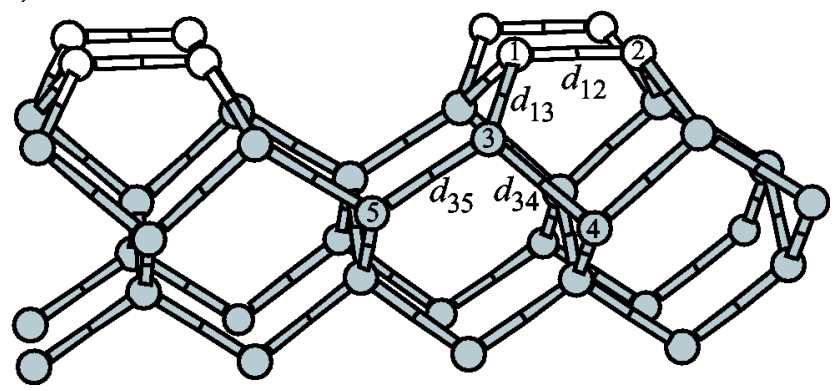

FIG. 6. Side view of (a) the $(2 \times 1)$-Pandey-reconstructed (111) surface and (b) the $(2 \times 1)$-reconstructed (001) surface of diamond, with indications of the distances occurring in Table IV. Threefold and fourfold coordinated atoms are drawn white and gray, respectively.

CBOP for these properties are at least as good as those of the REBO potential. In Fig. 5, $V_{i j}^{t o t}, \hat{E}_{i j}^{L R}$, and $F_{c, i j}$ are shown as functions of the interatomic distance, based on the discrete values for various coordination environments at the corresponding equilibrium nearest neighbor distances. This figure illustrates that the smooth dependence of these quantities on coordination, at the basis of the success of bond order potentials, is preserved when long-range interactions are added.

The bond order for the regular lattices, resulting from the fit of the radial part of the potential, fixes the angular function $G(\cos \theta)$ at the discrete points where $\cos \theta$ takes the values $-1,-1 / 2,-1 / 3,0$, and $1 / 2$, corresponding to the chain (ch), the graphite sheet (gr), diamond (d), the simple cubic (sc) and the fcc lattice. A continuous function $G$ is then constructed by a spline, based on these points and on the curvatures, i.e., the first and second derivatives of $G$ with respect to $\cos \theta$, at $\cos \theta=-1 / 2$ and $-1 / 3$ yielding the best possible fit of the elastic properties of the graphitic sheet and of diamond. The results are given in Table II, again compared with those of the Brenner I and the REBO potential. For diamond, the elastic constant for uniaxial compression, $c_{11}$, depends only on the second derivative of $G$ at $\cos \theta$ $=-1 / 3$, since the contributions from the first derivative cancel out in the summation over the bond angles in Eq. (8) for small uniaxial deformation. Fitting the elastic constant for shear, $c_{44}$, calculated including internal relaxations, fixes the first derivative at $\cos \theta=-1 / 3$. For the graphitic sheet, no exact agreement for both $c_{11}$ and $c_{66}$ (in-plane shear) could be obtained for the given form of the angular dependence in Eq. (8), but a quite good best fit resulted. Both for $c_{11}$ and $c_{66}$, internal relaxations were taken into account. It can be shown that the value of the first derivative of $G$ at $\cos \theta=$
TABLE IV. Surface energy (in eV) and interatomic distances (in $\AA$ ) of a relaxed $(2 \times 1)$-Pandey-reconstructed (111) surface (IVa) and a $(2 \times 1)$-reconstructed $(001)$ surface $(\mathrm{IVb})$ for the various potentials. The reference values are the results from $a b$ initio calculations (Ref. 43) and low-energy electron diffraction (LEED) experiments (values in brackets) (Ref. 32). The distances are indicated in Fig. 6.

\begin{tabular}{lccccc}
\hline \hline & Ref. & LCBOP & CBOP & Brenner I & REBO \\
\hline & \multicolumn{5}{c}{ (a) } \\
$E_{\text {surf }}$ & 1.87 & 1.28 & 1.44 & 1.05 & 1.01 \\
$d_{12}$ & 1.43 & 1.445 & 1.441 & 1.435 & 1.437 \\
$d_{13}$ & 1.54 & 1.539 & 1.539 & 1.547 & 1.559 \\
$d_{24}$ & 1.54 & 1.547 & 1.549 & 1.546 & 1.565 \\
$d_{35}$ & $1.61(1.62)$ & 1.622 & 1.633 & 1.605 & 1.621 \\
$d_{46}$ & $1.65(1.64)$ & 1.657 & 1.655 & 1.601 & 1.653 \\
& & & & & \\
& & & $(\mathrm{~b})$ & & \\
$E_{\text {surf }}$ & 2.12 & 2.31 & 2.50 & 1.71 & 2.14 \\
$d_{12}$ & 1.37 & 1.477 & 1.476 & 1.380 & 1.443 \\
$d_{13}$ & 1.50 & 1.542 & 1.548 & 1.513 & 1.556 \\
$d_{34}$ & 1.57 & 1.614 & 1.626 & 1.594 & 1.602 \\
$d_{35}$ & 1.55 & 1.543 & 1.538 & 1.506 & 1.555 \\
\hline \hline
\end{tabular}

$-1 / 3$, required to fit $c_{44}$ for diamond, depends on the first and second derivatives of the function $H\left(\delta r_{i j k}\right)$ around $\delta r_{i j k}=0$. These derivatives depend on one parameter, $C_{1}$, which was chosen such that the required curvature for $\mathrm{G}$ at $\cos \theta=-1 / 3$ permits a smooth spline of $G(\cos \theta)$ within the interval $\cos \theta \in[-1 / 2,-1 / 3]$, as illustrated in the inset of Fig. 1(a). Assuming a nonconstant function $H$ is the reason for the significantly improved shear elastic constant for diamond according to the LCBOP and CBOP in comparison to the REBO potential. The derivatives of $G$ at $\cos \theta=-1$ and 1 are set equal to zero and the remaining derivatives are obtained by finite difference expressions. The value of $G(1)$ is chosen is such a way that a smooth continuation of $G$ is obtained for $\cos \theta>1 / 2$. The resulting splines for the LCBOP and CBOP are shown in Fig. 1(a).

The interpolation for $F^{\text {conj }}$ (see the Appendix) is based on its values on the matrix of integer arguments $\left(N_{i j}, N_{j i}, N_{i j}^{c o n j}\right)$. The diagonal elements (i.e., $\left.N_{i j}=N_{j i}\right)$ corresponding to the regular lattices vanish. The values of $F^{c o n j}(2,3,0)=F^{c o n j}(2,3,1)$ and $F^{c o n j}(1,2,0)$ are determined by fitting the vacancy energies of diamond and graphite, equal to 7.2 (Ref. 34) and $7.6 \mathrm{eV},{ }^{35}$ respectively. The other values of $F^{c o n j}$ are fitted to available ab initio data or to the bond energies resulting from the REBO potential for appropriate cluster configurations. For example, the cluster in Fig. 4 was used to determine the value $F^{c o n j}(1,1,1)$ for the triple bond. In all cases, only the $i j$ bond is relaxed, whereas the bond angles and distances in the $i$ and $j$ "branches" are kept fixed at their ideal values corresponding to the coordinations $Z$ of the atoms within these branches, i.e., $\theta=180^{\circ}, 120^{\circ}$, and $109^{\circ}$, and $r_{c c}=1.325,1.420$, and $1.544 \AA$ for $Z=2,3$, and 4 respectively. All parameters of LCBOP and CBOP are listed in Tables III(a), III(b), and III(c). 


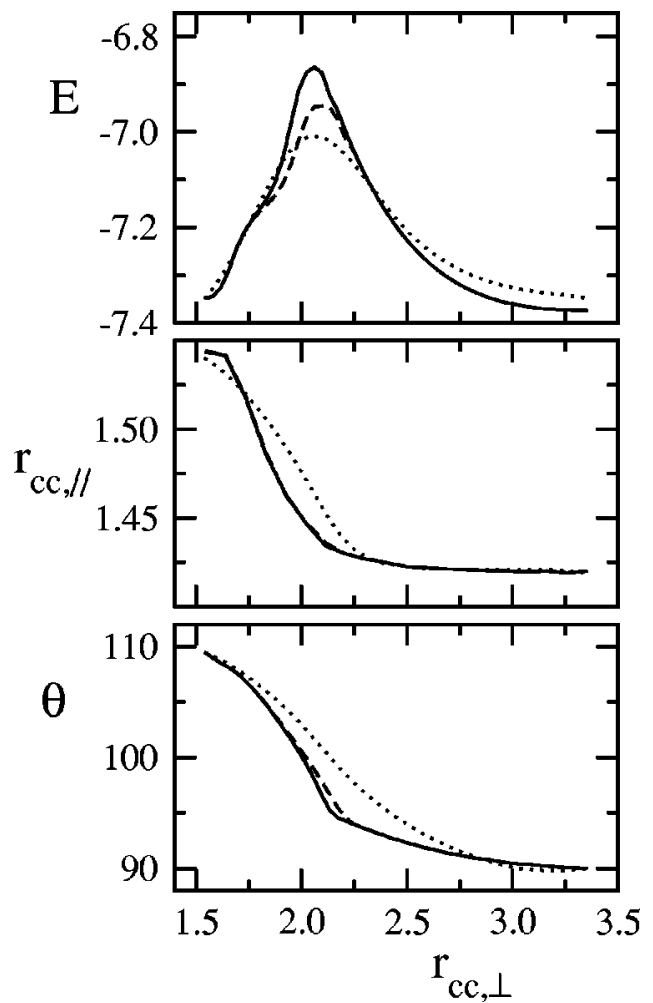

FIG. 7. The reaction path of the bulk diamond to graphite transformation as a function of the carbon-carbon distance perpendicular to the bilayers (transforming to graphitic layers), $r_{c c, \perp}$ (in $\AA$ ), for setting A (solid lines) and B (dashed lines), compared with the $a b$ initio results from Ref. 33 (dotted lines). The path is characterized by (a) the energy barrier (in eV), (b) the intraplanar carbon-carbon distance, $r_{c c, \perp}$ (in $\AA$ ), and (c) the buckling angle $\theta$ (in degrees).

The performances for the reconstructed (111) and (001) surfaces, shown in Figs. 6(a) and 6(b), are listed in Table IV. The description of the Pandey (111)- $(2 \times 1)$ surface is better than that of the (001)- $(2 \times 1)$ surface. The deviations with respect to the ab-initio results are similar to those of the REBO potential.

We have also calculated the formation energy of a typical defect in graphite, the so-called 5-77-5 defect, a topological defect which is formed by rotating one bond by $\pi / 2$ within a graphitic sheet, implying a transformation of four hexagons into two pentagons and two heptagons. A tight-binding calculation for this defect, which often occurs in nanotubes, resulted in a formation energy of $4.43 \mathrm{eV}^{36}$ We find formation energies equal to $4.41,4.98,2.58$, and $4.64 \mathrm{eV}$ for the LCBOP, CBOP, Brenner I, and REBO potentials respectively.

Particularly relevant for graphitization are the energetics and structural properties of the continuous transformation of diamond to rhombohedral graphite. Following Ref. 33, the total energy $E$ along this transformation can be expressed as a function of the interlayer bond length $r_{c c, \perp}$, the intralayer bond length $r_{c c, \|}$, and the buckling angle $\theta_{b}$ within the bilayers, i.e., $E=E\left(r_{c c, \perp}, r_{c c, \|}, \theta_{b}\right)$. A reaction path can be defined as the path yielding the minimal energy $E_{\text {min }}$ as a function of $r_{c c, \perp}$ varying from its value for diamond $(1.544 \AA$ ) to the equilibrium interlayer distance in graphite $(\sim 3.35 \AA)$. In Fig. 7, this reaction path according to the LCBOP is shown for two settings of the cutoff parameters of the short-range part of our potential, hereafter denoted as settings A and B and specified in Table III(a). There is a reasonable overall agreement with the $a b$ initio results from Ref. 33, represented by the dotted lines in Fig. 7. For setting B the height of the energy barrier is closer to the $a b$ initio result but the position of the maximum is shifted slightly to the right, as compared to the results for the preferred setting A. In our simulations we have used both settings for comparison.

Finally, to end the description of the potential, we want to mention that so far we have not included torsional interactions related to rotation about single and double bonds. Torsional interactions are particularly relevant for many hydrocarbon molecules. We expect their role in graphitization of pure diamond to be limited. However, if desired, torsional interactions in the style of those in Refs. 8 and 20, with possible modifications to meet recent $a b$ initio calculations of torsional energy barriers, ${ }^{37}$ can be added to the (L)CBOP without requiring a reparametrization. Furthermore, we have not included a correction for configurations with low coordination and small angles. Also this correction can be included relatively easily following the strategy used for the REBO potential. ${ }^{8}$

\section{GRAPHITIZATION OF DIAMOND}

We have performed simulations of diamond graphitization using the Monte Carlo technique for an $(N, P, T)$ ensemble. Trial moves are either accepted or rejected according to the Metropolis scheme, assuming Boltzmann statistics. ${ }^{38}$

We have performed annealing simulations for nonreconstructed and $(2 \times 1)$-Pandey-reconstructed (111) slabs of diamond. Several of the simulations using the LCBOP were done for both settings $\mathrm{A}$ and $\mathrm{B}$, in order to investigate the impact of the difference in the height of the barrier for the bulk graphite to bulk diamond transformation on surface graphitization. We have also focused our attention on the dependence of the graphitization process on the thickness of the slab. Note that in the AIMD studies of graphitization presented in the literature so far, ${ }^{24-26}$ the sample size is always relatively small due to computational limitations. Clearly, an empirical potential makes a study of size effects much easier.

In Fig. 8 three snaphots of a thin, nonreconstructed (111) slab are shown: the initial configuration and two configurations at a temperature of $1400 \mathrm{~K}$, using the LCBOP with setting A. From our annealing path we conclude that the transformation to perfect graphitic layers takes places at a temperature between 1300 and $1400 \mathrm{~K}$. The same simulation for the LCBOP with setting B also leads to perfect graphitic layers in qualitatively the same way, but now the transformation occurs between 1100 and $1200 \mathrm{~K}$. As expected, there is indeed a relation between the height of the barrier for the transformation of the bulk phases and the activation barrier for surface graphitization. The graphitization process is initiated at the surface. Once a graphitic nucleus has been formed within the surface bilayer, the graphitization starts to 
a)

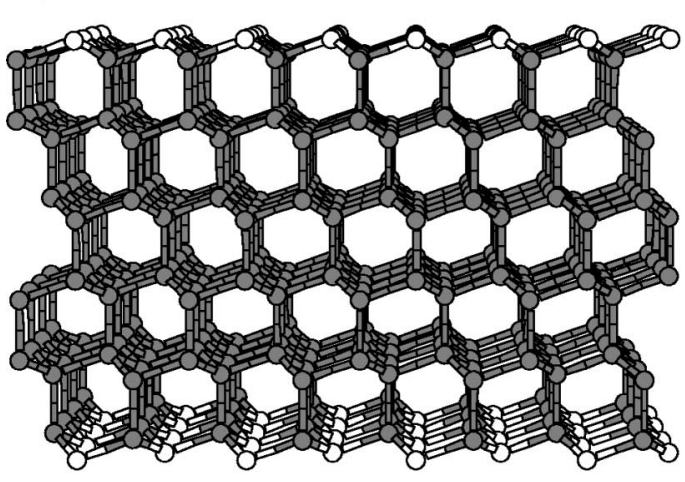

b)

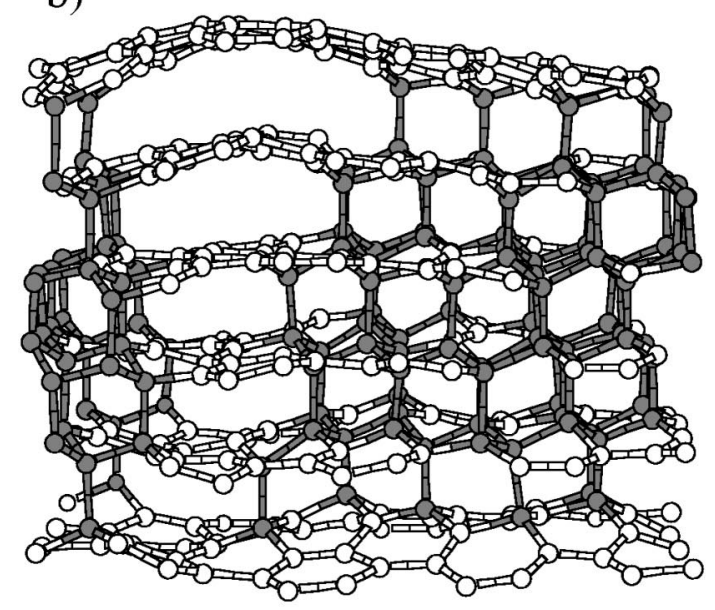

c)
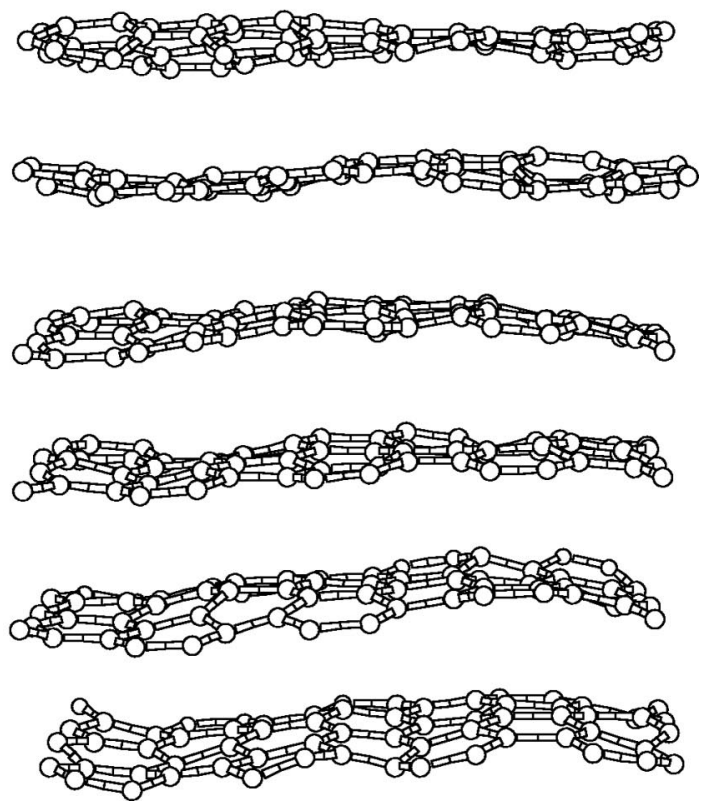

FIG. 8. Snapshots during a $(N, P, T)$ Monte Carlo annealing simulation of a thin diamond (111)-slab containing 384 atoms at (a) $0 \mathrm{~K}$ (initial configuration), (b) and (c) $1400 \mathrm{~K}$. White and gray balls are atoms with coordination three and four respectively. proceed perpendicular to the surface layer below this nucleus. This mechanism of perpendicular progression of the graphitization process was also observed in AIMD simulations $^{24,26}$ for thin slabs. In these simulations a rather low graphitization temperature was found for the nonreconstructed thin slabs, comparable to what we find.

If we take a thicker sample, the graphitization mechanism changes. Instead of the perpendicular progression mechanism we find a layer by layer mechanism. This is illustrated in Fig. 9, which shows snapshots of two simulations for unreconstructed (111) samples consisting of 12 bilayers, again using the LCBOP with setting A. The snapshot in Fig. 9(a), taken at $2250 \mathrm{~K}$, is from a simulation assuming a slab geometry. For the simulation of the snapshot in Fig. 9(b), taken at $2500 \mathrm{~K}$, a fixed substrate, simulated by not allowing moves in the lowest two bilayers, was assumed. In Fig. 9(a), we see traces of the $(2 \times 1)$-Pandey reconstruction, represented by the typical pentagonal shape at the right side between the second and third bilayers. The first complete graphitic layer was formed at $2000 \mathrm{~K}$ for both simulations. This graphitization temperature is more than $600 \mathrm{~K}$ higher than that for the thin slab in Fig. 8, which is another indication that a slab of only six bilayers is too small to be representative for a bulk with a surface. In part this is due to the strong conjugation effects, typical of carbon, requiring the extension beyond nearest neighbor represented by $F^{c o n j}$. Consequently, there is a strong surface-surface interaction for thin slabs. In contrast, the behavior for a slab with 12 bilayers seems to be representative for that of a single surface as indicated by the fact that both simulations of Fig. 9 show a layer by layer mechanism with the first graphitic layer appearing at the same temperature.

For each of the simulations we did for both settings of the LCBOP, including those for Pandey-reconstructed (111) surfaces, the graphitization temperature for setting $\mathrm{A}$ was between 100 and $200 \mathrm{~K}$ lower than that for setting B. Although a correspondence exists, this difference in graphitization temperature is much less than the difference in the heights of the barriers for the bulk transformation, which is $\sim 70 \mathrm{meV}$ $(\sim 800 \mathrm{~K})$.

The same difference of the graphitization mechanism for thin and thick samples was also observed for $(2 \times 1)$-Pandey-reconstructed (111) slabs. Typically, once a few bonds between the first two bilayers are broken, the whole lower zigzag chain rapidly detaches from the surface, leading to curved graphitic strips at the surface as shown in Fig. 10 (compare with Fig. 6). The graphitization temperatures are higher than for the non-reconstructed slabs, being equal to $\sim 2500 \mathrm{~K}$ for a six-bilayers slab and $\sim 2750 \mathrm{~K}$ for a 12-bilayer slab using the LCBOP with setting A. These transition temperatures are lower than the $\sim 3500 \mathrm{~K}$ found in the AIMD simulations of Ref. 26 for a reconstructed thin slab. This difference could be explained by the difference between the MC and MD techniques. Although there is no real "time scale" in MC simulations, a rough time correspondence can be established via the phonon frequencies. Atomic movements occur on pico- to nanosecond time scale. So our MC simulation, with typically a 100000 moves per atoms, would correspond to $10^{-7}-10^{-4} \mathrm{sec}$, which is much larger than the 


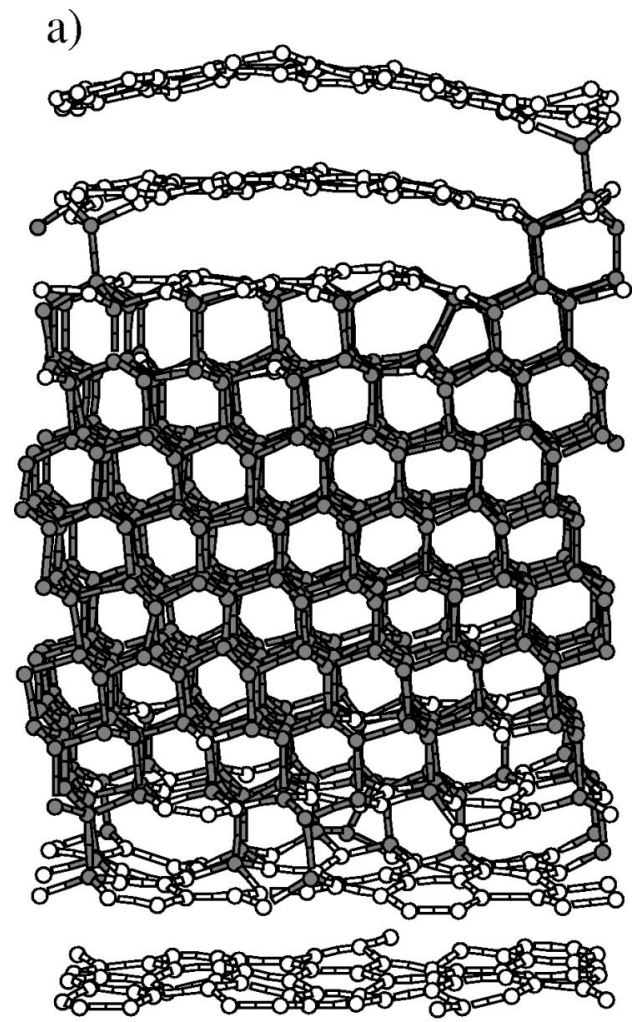

b)

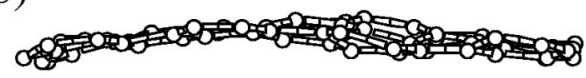

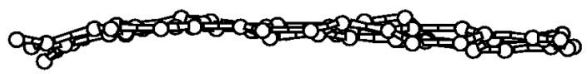
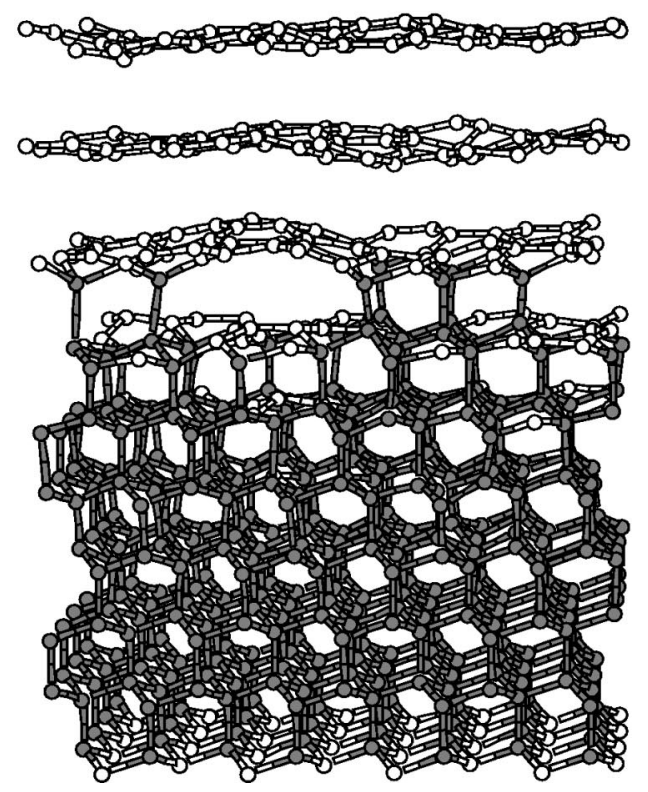

FIG. 9. Snapshots during a $(N, P, T)$ Monte Carlo annealing simulation at 2250 and $2500 \mathrm{~K}$ for respectively (a) a "thick" diamond (111) slab containing 768 atoms and (b) a diamond (111) surface with a fixed substrate, allowing no moves in the lowest two bilayers. Color code as is Fig. 8.

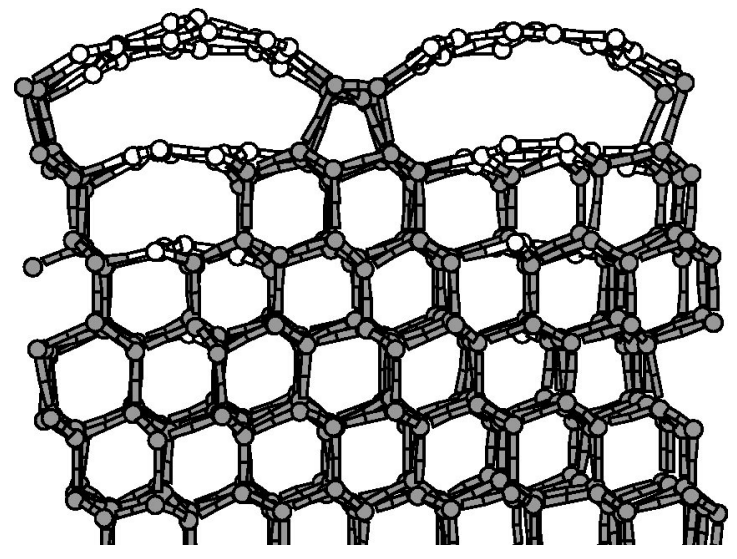

FIG. 10. Snapshot during a $(N, P, T)$ Monte Carlo annealing simulation at $2500 \mathrm{~K}$ of the upper six bilayers of $(2 \times 1)$-Pandey-reconstructed (111) surface with 768 atoms, assuming a fixed substrate. Color code as is Fig. 8.

typical time scale of picoseconds to nanoseconds in MD simulations. Clearly, this longer "time scale" in MC simulation yields a larger probability to see structural changes. Furthermore, the probability to overcome a barrier for the breaking (or formation) of a bond is typically larger for $\mathrm{MC}$ simulations than for MD simulations, especially when the maximal displacement of the atoms, chosen to be temperature dependent as to yield acceptance percentages between $30 \%$ and $50 \%,{ }^{38}$ becomes comparable to the width of the barrier.

To compare with an alternative approach for the longrange extension in the spirit of previous work $^{20,21}$ we have done simulations for the thin nonreconstructed slab also for extended Brenner I and extended REBO potentials. In these extensions, the total energy is written as in Eq. (1), but with the long-range potential represented by the dashed line in Fig. 2(a) and a different switching function $S_{i j}$. To avoid interference with the short-range potential, long-range interactions up to third nearest neighbors are excluded, which is accomplished by taking

$$
S_{i j}=\left(1-f_{i j}\right) \prod_{k}\left(1-f_{i k} f_{k j}\right) \prod_{k, l}\left(1-f_{i k} f_{k l} f_{l j}\right)
$$

yielding continuous derivatives with respect to the atomic positions for any configuration. The results are shown in Figs. 11(a) and 11(b). Whereas the extended REBO potential gives rise to the formation of a more or less graphitic structure, but with defects, the structure resulting from the extended Brenner I potential is $s p^{2}$-hybridized amorphous. The reason for this remarkable difference is not so clear. It may either be due to the improved elastic properties of REBO, or to the extension in the description of the conjugation correction for REBO. ${ }^{8}$ Considering the coordination changes occurring during the process, it is clear that a certain subset of the matrix elements of $F^{c o n j}$, and also the interpolation of 
a)

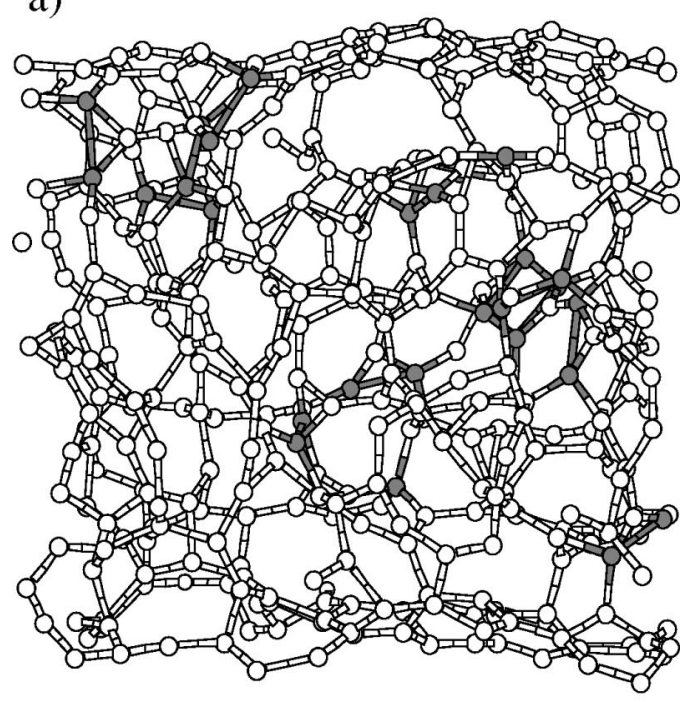

b)

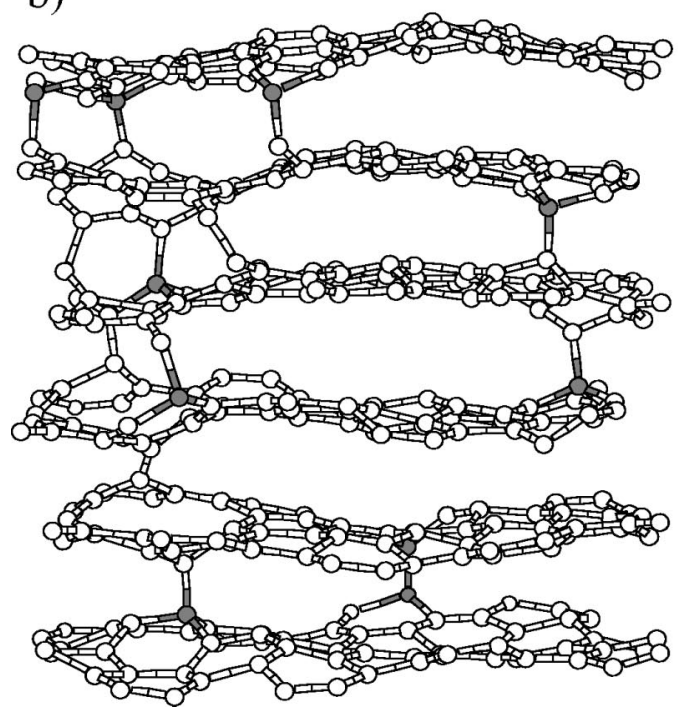

FIG. 11. Snapshots during a $(N, P, T)$ Monte Carlo annealing simulation for a thin (111) slab at 3500 and $3000 \mathrm{~K}$ using respectively (a) an extended Brenner I potential and (b) an extended REBO potential. Color code as is Fig. 8.

$F^{c o n j}$ for the various possible coordination configurations intermediate between $s p^{3}$ and $s p^{2}$, play important roles in graphitization. This may actually also be the reason why the extended REBO potential gives defects [see Fig. 11(b)] which do not disappear for simulations up to $3500 \mathrm{~K}$. The LCBOP, with our interpolation scheme for $F^{c o n j}$, leads instead to perfect graphitic layers in agreement with AIMD simulations, at least for samples of this size.

Apart from these structural differences, another important difference is the temperature at which the structural changes take place, which is much higher for both extended Brenner potentials than for the LCBOP, apparently due to too high barriers for bond breaking (and formation).

To conclude, we show the results of an annealing simulation starting from a diamond wedge, containing 2808 atoms,
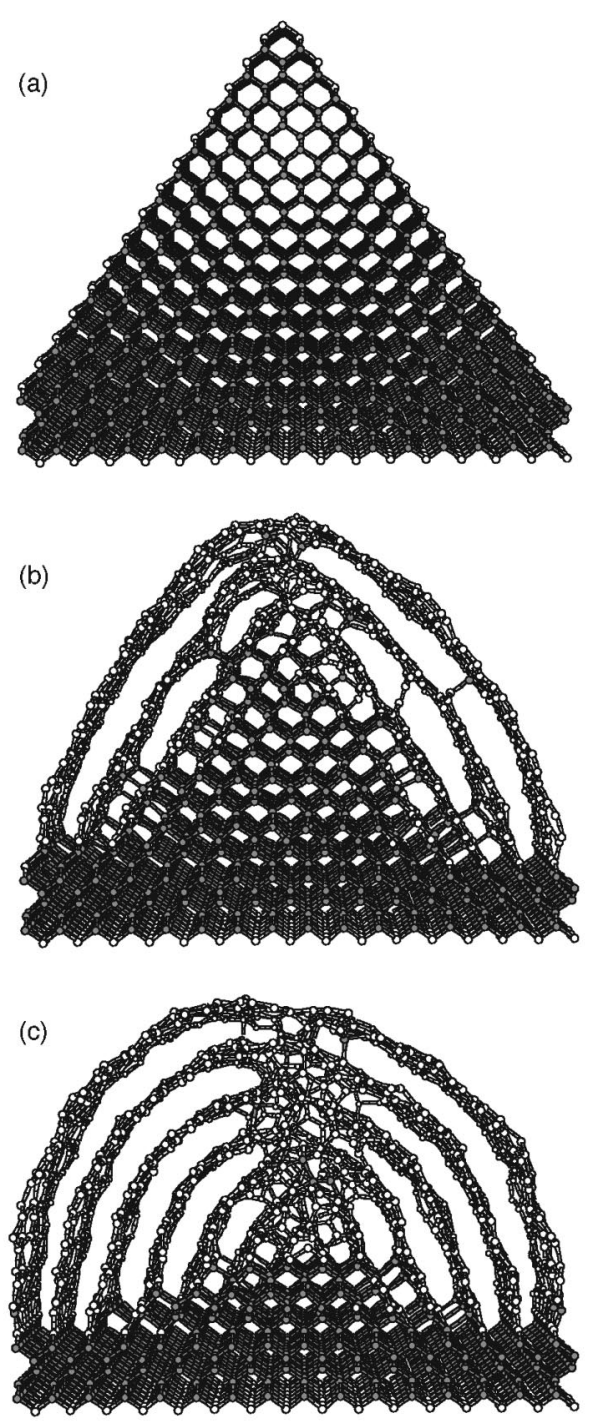

(d)

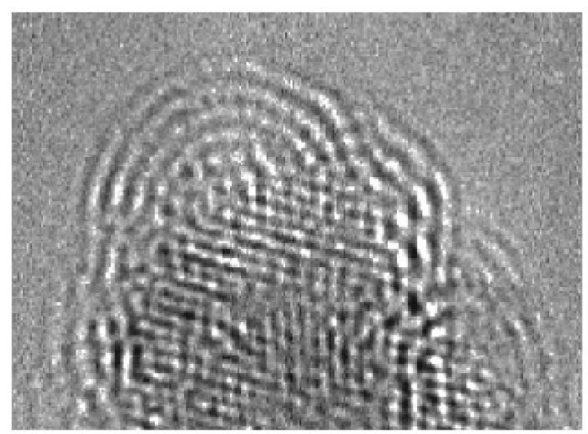

$2 \mathrm{~nm}$

FIG. 12. Snapshots during a $(N, P, T)$ Monte Carlo annealing simulation of a diamond wedge containing 2808 atoms, bounded by nonreconstructed $\{111\}$ planes, at (a) $0 \mathrm{~K}$ (starting configuration), (b) $2500 \mathrm{~K}$, and (c) $3000 \mathrm{~K}$. Color code as is Fig. 8. The very few twofold coordinated atoms are indicated as white balls, as the threefold coordinated atoms. For comparison, (d) shows a high resolution electron microscopy image of irradiated diamond (Ref. 39). The original $\{111\}$ orientations of the diamond structure are still visible as straight lines in the inner bulk region. 
bounded by nonreconstructed $\{111\}$ planes, as shown in Fig. 12(a). The two lowest bilayers are held fixed, to simulate the diamond bulk. Periodicity was assumed in the $y$ direction perpendicular to the images. The two shapshots in Figs. 11(b) and 11(c), taken at 2500 and $3000 \mathrm{~K}$, respectively, show the formation of a shell-like graphitic structure, albeit with defects around the $x=0$ plane, i.e., the vertical plane perpendicular to the image. The graphitized layer segments are pushed outwards, to minimize the interlayer energy. For the given geometry, with a fixed substrate, this leads to concentric shells, which strongly reminds a transmission electron micrograph of a protrusion on the surface of irradiated diamond, ${ }^{39}$ as shown in Fig. 12(d). In Ref. 39 the distance between the graphitic layers close to the diamond bulk and that between the outer planes are reported to be 2.9 and 3.4 $\AA$, respectively. From our simulation we find these distances to be equal to 2.91 and $3.44 \AA$, providing a very nice illustration of the capabilities of our potential.

\section{SUMMARY AND PERSPECTIVES}

We have constructed an intrinsic long-range bond order potential for carbon (LCBOP), which smoothly bridges the gap between the strong covalent and the weak intermolecular interactions, important for bond breaking and formation. The LCBOP is an appropriately parametrized mix of a shortrange Brenner-like bond order potential and a long-range, radial potential. Besides accurate values for bond distances, binding energies and stretching force constants for a large set of coordination environments, it gives (i) good elastic constants for diamond and graphite, (ii) a reasonable description of the reaction path for the bulk diamond to graphite transition, as well as (iii) a good description of the interlayer interaction energy in graphite over a range of interlayer distances, as compared to experimental and/or ab initio data.

In this work, we have studied, by means of extensive MC simulations, the process of graphitization at surfaces in several geometries. For thin (111) slabs, we find a structural path for the graphite to diamond transformation which compares well with AIMD simulations. However a layer by layer graphitization is found for thicker slabs. Simulations for wedge geometries lead to multishell structures, giving a realistic picture in comparison to experimental observations, with in addition a good prediction of the distances between the graphitic layers in this nonplanar geometry.

Besides the LCBOP we have also presented a short-range potential, CBOP. Both the LCBOP and CBOP have good elastic properties, with in particular a much more accurate shear elastic constant for diamond as compared to that of Brenner's REBO potential, ${ }^{8}$ which makes them good candidates for simulations of large diamond systems subject to strain, with possible applications, among others, for diamond coatings. ${ }^{40,41}$ However, the main contribution of this work to the field of carbon-based materials is the careful inclusion of long-range interactions, making the LCBOP very suitable to study the structural properties of (multishell) fullerenes and nanotubes.

\section{ACKNOWLEDGMENTS}

This work was financed by NWO (Nederlandse Organisatie voor Wetenschappelijke Onderzoek), Project No. 015.000.031. We are grateful to Dr. G. de Wijs for doing the ab initio LDA calculation of the interlayer energy in graphite, to Frank van Bouwelen for allowing publication in this paper of the experimental data shown in Fig. 12(d), and to A. Petukhov for his help in the initial stage of this work.

\section{APPENDIX}

We have constructed an analytic interpolation for the bivariable function $F_{N_{i j}^{c o n j}}^{c o n j}\left(N_{i j}, N_{j i}\right)$, which is based on the function values and derivatives at the grid of integer $\left(N_{i j}, N_{j i}\right)$. The derivatives at the grid points have to be determined according to the following prescriptions. Due to symmetry the derivatives with respect to the two variables on the grid points $(n, m)$ and $(m, n)$ are related by

$$
\left.\frac{\partial F_{N_{i j}^{c o n j}}^{c o n j}}{\partial N_{i j}}\right|_{n, m}=\left.\frac{\partial F_{N_{i j}^{c o n j}}^{c o n j}}{\partial N_{j i}}\right|_{m, n} .
$$

Continuity at the boundaries implies

$$
\left.\frac{\partial F_{N_{i j}^{c o n j}}^{c o n j}}{\partial N_{i j}}\right|_{0, m}=\left.\frac{\partial F_{N_{i j}^{c o n j}}^{c o n j}}{\partial N_{i j}}\right|_{3, m}=0 .
$$

For $n=1$ or 2 , if the value of $F_{N_{i j}^{c o n j}}^{c o n j}(n, m)$ lies in between that of $F_{N_{i j}^{c o n j}}^{c o n j}(n+1, m)$ and $F_{N_{i j}^{c o n j}}^{c o n j}(n-1, m)$, the derivative is given by the finite difference expression

$$
\left.\frac{\partial F_{N_{i j}^{c o n j}}^{c o n j}}{\partial N_{i j}}\right|_{n, m}=\frac{F_{N_{i j}^{c o n j}}^{c o n j}(n+1, m)-F_{N_{i j}^{c o n j}}^{c o n j}(n-1, m)}{2},
$$

else it is set to zero to avoid extrema in between two grid points. The prescription is completed by a few exceptions, related to avoiding oscillations. These are

$$
\left.\frac{\partial F_{1}^{c o n j}}{\partial N_{i j}}\right|_{2,0}=-0.088188
$$

for LCBOP, and

$$
\begin{aligned}
& \left.\frac{\partial F_{0}^{c o n j}}{\partial N_{i j}}\right|_{2,0}=-0.072300, \\
& \left.\frac{\partial F_{1}^{c o n j}}{\partial N_{i j}}\right|_{2,0}=-0.052143
\end{aligned}
$$

for CBOP.

For convenience, in the further description we will omit the subscript argument $N_{i j}^{c o n j}$, which is either 0 or 1 . For arbitrary real values of $\left(N_{i j}, N_{j i}\right)$, the value of $F^{c o n j}$ is given by the interpolation within the square to which the point $\left(N_{i j}, N_{j i}\right)$ belongs. We denote the interpolation within this 
square as $f^{c o n j}(x, y)$ where $x$ and $y$ are numbers between 0 and 1 defined by $x \equiv N_{i j}-\operatorname{Int}\left(N_{i j}\right)$ and $y \equiv N_{j i}-\operatorname{Int}\left(N_{j i}\right)$. The interpolation is given by

$$
\begin{aligned}
f^{c o n j}(x, y)= & (1-y)(1-x)\left[f_{00}^{c o n j}+x^{2} \widetilde{f}_{x, 10}+y^{2} \widetilde{f}_{y, 01}\right] \\
& +(1-y) x\left[f_{10}^{c o n j}+(1-x)^{2} \widetilde{f}_{x, 00}+y^{2} \widetilde{f}_{y, 11}\right] \\
& +y(1-x)\left[f_{01}^{c o n j}+x^{2} \widetilde{f}_{x, 11}+(1-y)^{2} \widetilde{f}_{y, 00}\right] \\
& +x y\left[f_{11}^{c o n j}+(1-x)^{2} \widetilde{f}_{x, 01}+(1-y)^{2} \widetilde{f}_{y, 10}\right],
\end{aligned}
$$

where:

$$
\begin{aligned}
& \tilde{f}_{x, k l}=(-1)^{k}\left(\left.\frac{\partial f^{c o n j}}{\partial x}\right|_{k l}-f_{1 l}^{c o n j}+f_{0 l}^{c o n j}\right), \\
& \tilde{f}_{y, k l}=(-1)^{l}\left(\left.\frac{\partial f^{c o n j}}{\partial y}\right|_{k l}-f_{k 1}^{c o n j}+f_{k 0}^{c o n j}\right),
\end{aligned}
$$

with $k, l=0,1, f_{k l}^{c o n j} \equiv f^{c o n j}(k, l)$, and $\partial f^{c o n j} /\left.\partial \alpha\right|_{k l}(\alpha=x, y)$ are the derivatives of $F^{c o n j}$ in the corners $(k, l)$. Finally, we note that $F_{i j}^{c o n j}$ is continuous everywhere up to the first derivatives with respect to the atomic positions, due to the fact that both $\partial F^{\text {conj }} /\left.\partial N_{i j}\right|_{N_{i j}=3}=0$ and $\partial F^{c o n j} /\left.\partial N_{i j}^{c o n j}\right|_{N_{i j}=3}$ $=0$.
${ }^{1}$ F.H. Stillinger and T.A. Weber, Phys. Rev. B 31, 5262 (1985).

${ }^{2}$ M.Z. Bazant, E. Kaxiras, and J.F. Justo, Phys. Rev. B 14, 8542 (1997).

${ }^{3}$ J.F. Justo, M.Z. Bazant, E. Kaxiras, V.V. Bulatov, and S. Yip, Phys. Rev. B 5, 2539 (1998).

${ }^{4}$ N.A. Marks, Phys. Rev. B 63, 035401 (1997).

5 J. Tersoff, Phys. Rev. Lett. 56, 632 (1986); Phys. Rev. B 37, 6991 (1988); 38, 9902 (1988).

${ }^{6}$ J. Tersoff, Phys. Rev. Lett. 25, 2879 (1988).

${ }^{7}$ D.W. Brenner, Phys. Rev. B 42, 9458 (1990).

${ }^{8}$ D.W. Brenner, O.A. Shenderova, J.A. Harrison, S.J. Stuart, B. Ni, and S.B. Sinnott, J. Phys.: Condens. Matter 14, 783 (2002).

${ }^{9}$ M.W. Finnis and J.E. Sinclair, Philos. Mag. A 50, 45 (1984).

${ }^{10}$ M.I. Baskes, Phys. Rev. Lett. 23, 2666 (1987); M.I. Baskes, J.S. Nelson, and A.F. Wright, Phys. Rev. 40, 6085 (1989).

${ }^{11}$ J. Cai and J.S. Wang, Phys. Rev. B 64, 035402 (1999).

${ }^{12}$ D.G. Pettifor, Phys. Rev. Lett. 22, 2480 (1989); D.G. Pettifor and I.I. Oleinik, Phys. Rev. B 59, 8487 (1999).

${ }^{13}$ I.I. Oleinik and D.G. Pettifor, Phys. Rev. B 59, 8500 (1999).

${ }^{14}$ B.J. Thijsse, Phys. Rev. B 65, 195207 (2002).

${ }^{15}$ L.A. Girifalco and R.A. Ladd, J. Chem. Phys. 25, 693 (1956).

${ }^{16}$ D.P. DiVicenzo, E.J. Mele, and N.A.W. Holzwarth, Phys. Rev. B 27, 2458 (1983).

${ }^{17}$ S.B. Sinnott, O.A. Shenderova, C.T. White, and D.W. Brenner, Carbon 36, 1 (1998).

${ }^{18}$ A. Petukhov and A. Fasolino, Technical Proceedings of the ICCN 2001 International Conference on Computational Nanoscience, Applied Computational Research Society (also at http:// www.cr.org/publications/ICCN2001/).

${ }^{19}$ J. Che, T. Cagin, and W.A. Goddard III, Theor. Chem. Acc. 102, 346 (1999).

${ }^{20}$ S.J. Stuart, A.B. Tutein, and J.A. Harrison, J. Chem. Phys. 112, 6472 (2000).

${ }^{21}$ J.H. Los and A. Fasolino, Comput. Phys. Commun. 147, 178 (2002)

${ }^{22}$ V.L. Kuznetsov, I.L. Zilberberg, Yu.V. Butenko, A.L. Chuvilin, and B. Segall, J. Appl. Phys. 86, 863 (1999).

${ }^{23}$ M. Seal, Nature (London) 185, 522 (1960).
${ }^{24}$ A. De Vita, G. Galli, A.C. Canning, and R. Car, Nature (London) 379, 523 (1996).

${ }^{25}$ G. Jungnickel, D. Porezag, T. Frauenheim, M.I. Heggie, W.R.L. Lambrecht, B. Segall, and J.C. Angus, Phys. Status Solidi A 154, 109 (1996).

${ }^{26}$ G. Kern and J. Hafner, Phys. Rev. B 58, 13167 (1998).

${ }^{27}$ G.C. Abell, Phys. Rev. B 31, 6184 (1985).

${ }^{28}$ R. Nicklow, W. Wakabayashi, and H.G. Smith, Phys. Rev. B 5, 4951 (1972).

${ }^{29}$ S.G. Kabalkina and L.F. Vereschagin, Dokl. Akad. Nauk. SSSR 131, 300 (1960) [Sov. Phys. Dokl. 5, 373 (1960)].

${ }^{30}$ Semiconductors: Physics of Group IV Elements and III-V Compounds, edited by O. Madelung, M. Schulz, and H. Weiss, Landolt-Börnstein, New Series, Group III, Vol. 17, Pt. a (Springer, Verlag, Berlin, 1982).

${ }^{31}$ A. Aziz, A. Rafizadeh, and H.A. Rafizadeh, Phys. Rev. B 7, 4527 (1973).

${ }^{32}$ S. Walter, J. Bernhardt, U. Starke, K. Heinz, F. Maier, J. Ristein, and L. Ley, J. Phys.: Condens. Matter 14, 3085 (2002).

${ }^{33}$ S. Fahy, S.T. Louie, and M.L. Cohen, Phys. Rev. B 34, 1191 (1986).

${ }^{34}$ J. Bernholc, A. Antonelli, T.M. Del Sole, Y. Bar-Yam, and S.T. Pantelides, Phys. Rev. Lett. 61, 2689 (1988).

${ }^{35}$ E. Kaxiras and K.C. Pandey, Phys. Rev. Lett. 61, 2693 (1988).

${ }^{36}$ B.C. Pan, W.S. Yang, and Jinlong Yang, Phys. Rev. B 62, 12652 (2000).

${ }^{37}$ C.J. Wu, J.N. Glosli, G. Galli, and F.H. Ree, Phys. Rev. Lett. 89, 135701 (2002).

${ }^{38}$ See, e.g., D. Frenkel and B. Smit, Understanding Molecular Simulation (Academic Press, San Diego, 1996), p. 2002.

${ }^{39}$ F.M. van Bouwelen, J.E. Field, and L.M. Brown (unpublished).

${ }^{40}$ J.W. Ager III and M.D. Drory, Phys. Rev. B 48, 2601 (1993).

${ }^{41}$ J.G. Buijnsters, P. Shankar, W. Fleischer, W.J.P. van Enckevort, J.J. Schermer, and J.J. ter Meulen, Diamond Relat. Mater. 11, 536 (2002).

${ }^{42}$ M.T. Yin and M.L. Cohen, Phys. Rev. Lett. 50, 2006 (1983); Phys. Rev. B 29, 6996 (1984).

${ }^{43}$ A. Scholze, W.G. Schmidt, and F.F. Bechstedt, Phys. Rev. B 53, 13725 (1996). 